\title{
On the Localization of Discontinuities of the First Kind for a Function of Bounded Variation
}

\author{
A. L. $\operatorname{Ageev}^{1,2}$ and T. V. Antonova ${ }^{1}$ \\ Received June 2, 2011
}

\begin{abstract}
Methods of the localization (detection of positions) of discontinuities of the first kind for a univariate function of bounded variation are constructed and investigated. Instead of an exact function, its approximation in $L_{2}(-\infty,+\infty)$ and the error level are known. We divide the discontinuities into two sets, one of which contains discontinuities with the absolute value of the jump greater than some positive $\Delta^{\mathrm{min}}$; the other set contains discontinuities satisfying a smallness condition for the value of the jump. It is required to find the number of discontinuities in the former set and localize them using the approximately given function and the error level. Since the problem is ill-posed, regularizing algorithms should be used for its solution. Under additional conditions on the exact function, we construct regular methods for the localization of discontinuities and obtain estimates for the accuracy of localization and for the separability threshold, which is another important characteristic of the method. The (order) optimality of the constructed methods on the classes of functions with singularities is established.

Keywords: ill-posed problem, discontinuity of the first kind, localization of singularities, regularizing algorithm.
\end{abstract}

DOI: $10.1134 /$ S0081543813020028

\section{INTRODUCTION}

Problems of localization of singularities belong to the class of nonlinear ill-posed problems, and regularizing algorithms should be used for their solution (the classical theory of ill-posed problems is presented, e.g., in [1-3]). These problems with different singularities ( $\delta$-functions, discontinuities of the first kind, or breaks) have a long history (see, for example, review [4]). Referring the reader to this review, we only briefly describe the localization of discontinuities of the first kind.

In [5], examples of medical problems can be found in which it is required to localize discontinuities of the first kind of a scalar function (signal). The position of a discontinuity is interpreted as a point of the independent variable corresponding to a jump of the "mode" of activity of an organism under consideration. For references to problems of localization of discontinuities of the first kind that arise in the engineering, see [6]. In the present paper, we consider only functions of one variable. Nevertheless, evidently, the case of one variable is the first step to the two-dimensional problem of localization of a line $[7,8]$ where a function has a discontinuity of the first kind. Boundaries of

\footnotetext{
${ }^{1}$ Institute of Mathematics and Mechanics, Ural Branch of the Russian Academy of Sciences, ul. S. Kovalevskoi 16, Yekaterinburg, 620990 Russia

emails: ageev@imm.uran.ru, tvantonova@imm.uran.ru

${ }^{2}$ Institute of Mathematics and Computer Science, Ural Federal University, pr. Lenina 51, Yekaterinburg, 620000 Russia
} 
many objects in images are such lines. A discussion of realistic models of optical images can be found, for example, in [9].

We pass to theoretical results on regularization, which, as far as the authors know, have been obtained for problems of localization of discontinuities of the first kind only for univariate functions and for the case of a finite number of discontinuities of the first kind; the function is assumed to be smooth outside the discontinuities.

All papers are naturally divided into two groups. In the first group (see, for example, [10,11]), the problem of localization of discontinuities of the first kind of a noisy univariate function is considered in the statistical formulation. The main results consist in constructing localization methods and in obtaining statistical upper estimates for the accuracy of localization of discontinuities.

The other large cycle of papers [12-17] (see also [4]) on the localization of singularities was implemented for problems in the deterministic formulation. In these papers, it is assumed that an exact function $x$ belongs to $L_{2}(-\infty,+\infty)$ (or $L_{p}(-\infty,+\infty), 1<p<\infty$ ), has a finite number of discontinuities of the first kind, and is smooth outside the discontinuities. In the meantime, in some applications, for example, in signal processing, it is natural to assume that the function $x$ does not decrease at $\pm \infty$, i.e., does not belong to $L_{p}(-\infty,+\infty)$ and has countably many discontinuities of the first kind. Therefore, it is reasonable to investigate other classes of functions with countably many singularities and carry over the methods of [12-17] to this case. As in [12-17], we assume that, instead of the exact function $x$, the error level $\delta$ and an approximate function $x^{\delta}$ are known such that $\left\|x-x^{\delta}\right\|_{L_{2}(-\infty,+\infty)} \leq \delta$. Since the disturbance $x-x^{\delta}$ is connected with the measuring apparatus (and the experimental conditions) and is independent of the properties of the exact function $x$, to our mind, such a statement has a right to exist.

In contrast to [12-17], in the present paper, we substantially use facts from the theory of the Lebesgue-Stieltjes integral [18], which allows us to justify the main decomposition for an auxiliary function by references to known results. Note also that [17] is the key paper that enabled us to consider the new statement. In this paper, a new law for the choice of the regularization parameter and new estimation methods are proposed, which allow us to consider countably many discontinuities of the first kind.

In the present paper, we construct and study methods for the localization (detection of positions) of discontinuities of the first kind for a function with countably many discontinuities. The discontinuities are divided into two sets, one of which contains discontinuities with the absolute value of the jump greater than some positive $\Delta^{\text {min }}$; the other set contains discontinuities satisfying a smallness condition for the value of the jump. It is required to find the number of discontinuities in the former set and localize them using the approximately given function and the error level. We construct regular methods for the localization of discontinuities. Under additional conditions on the exact function, we obtain estimates for the accuracy of localization and for the separability threshold, which is another important characteristic of the method. The (order) optimality of the constructed methods on the classes of functions with singularities is established.

Note some specificities of the presentation. The general scheme constructed in Section 2 allows us to obtain specific methods of localization in different situations; this is convenient for the reader familiar with basic methods. However, a large number of variants creates additional difficulties at the first reading. In addition, the most general conditions (close to necessary) on the exact function, which guarantee the efficiency of the proposed methods, have no simple and illustrative interpretation. Therefore, for the convenience of the reader, the authors voluntarily present the material under conditions that are clear though not most general. In addition, in 
Section 3 (see Corollaries 1-3), we consider simpler special cases of general statements (specific averaging functions, simpler conditions on the exact function, etc.), which can be obtained from the general scheme. In the same section, we give lower estimates for the accuracy of localization of discontinuities and the separability threshold. The combination of these estimates and the upper estimates obtained in Section 2 allows us to establish the order optimality of the constructed methods on the corresponding classes of functions with singularities.

\section{AUXILIARY STATEMENTS}

Let us agree that all the integrals considered in this paper are the Lebesgue-Stieltjes integrals [18]. We consider functions $x$ that are sufficiently smooth except for discontinuities of the first kind at points $s_{i}, i=1,2, \ldots$, and have limits $x\left(s_{i}+0\right)$ and $x\left(s_{i}-0\right)$. Denote by $\Delta_{i}=x\left(s_{i}+0\right)-x\left(s_{i}-0\right)$ the jump of the function $x$ at the point $s_{i}$. Denote by $[a, b]$ a finite closed interval. Assume that the interval $[a, b]$ is such that the interval $(a, b)$ contains no discontinuity points. In the case when one or both end-points of the interval coincide with discontinuity points $s_{i}$ of the function, we will take the liberty to say that $x$ is absolutely continuous on $[a, b]$ if it is redefined at the points $a$ and $b$ by continuity, i.e., by the values $x(a+0)$ and $x(b-0)$, respectively.

We define a linear space $M V$ of functions $x$ that satisfy the following conditions:

(i) the function $x$ has a finite number of discontinuities of the first kind in any interval $[a, b]$ and $\sum_{k=1}^{\infty}\left|\Delta_{k}\right|<\infty$;

(ii) the function $x$ is bounded on $(-\infty,+\infty)$;

(iii) the function $x$ is absolutely continuous on any interval $[a, b]$ such that the interval $(a, b)$ contains no discontinuity points;

(iv) the function $x^{\prime}$ is bounded almost everywhere ${ }^{3}$ on $(-\infty,+\infty)$.

By condition $(i)$, any function $x \in M V$ has at most countably many discontinuities of the first kind. As follows from conditions $(i)$ and (iii), the function $x$ has bounded variation on any interval $[a, b]$. Then, by $[18$, Ch. 8 , Sect. 3], the following decomposition of the function $x$ is valid:

$$
x(s)=x_{0}(s)+\sum_{i=1}^{\infty} \Delta_{i} \times \chi\left(s-s_{i}\right), \quad \chi(s)= \begin{cases}0, & s<0 \\ 1, & s \geq 0\end{cases}
$$

where $x_{0}$ is a continuous function.

Lemma 1. If $x \in M V$, then the function $x_{0}$ in decomposition (1.1) is absolutely continuous on any interval $[a, b]$.

Proof. We prove the lemma for the case of one discontinuity point $s_{1}$. In the general case, the proof is similar. Consider the integral with varying upper limit

$$
\hat{x}(s)=\int_{a}^{s} x_{0}^{\prime}(\tau) d \tau
$$

If we show that $\hat{x}(s)=x_{0}(s)-x_{0}(a)$, then, according to $\left[18\right.$, Ch. 9, Sect. 4], the function $x_{0}$ is absolutely continuous on $[a, b]$. For $s \leq s_{1}$, this follows from the fact that $x_{0}(\tau)=x(\tau)$ for $\tau \in[a, s]$.

\footnotetext{
${ }^{3}$ By conditions $(i)$ and $($ iii $)$, the derivative $x^{\prime}$ exists almost everywhere; at the points where it does not exists, we redefine this function, for example, by zero.
} 
Let $s>s_{1}$. Since $x(\tau)=x_{0}(\tau)$ for $\tau \in\left[a, s_{1}\right], x(\tau)-x_{0}(\tau)=\Delta_{1}$ is constant for $\tau \in\left[s_{1}, s\right]$, and the restriction of $x(\tau)$ to $\left[s_{1}, s\right]$ is an absolutely continuous function, we have

$$
\hat{x}(s)=\int_{a}^{s_{1}} x_{0}^{\prime}(\tau) d \tau+\int_{s_{1}}^{s} x_{0}^{\prime}(\tau) d \tau=\left(x_{0}\left(s_{1}\right)-x_{0}(a)\right)+\left(x_{0}(s)-x_{0}\left(s_{1}\right)\right)=x_{0}(s)-x_{0}(a) .
$$

The lemma is proved.

Throughout the paper, we will consider functions $x \in M V$. However, to make the presentation complete, we give two new conditions $\left(i i^{\prime}\right)$ and $\left(i^{\prime}\right)$. Let us agree that break points of a function are, by definition, discontinuity points of the first kind of its derivative.

Recall that (see [18, Ch. 5, Sect. 5; Ch. 9, Sect. 4]), if a function $x(s)$ defined on an interval $[a, b]$ has a derivative $x^{\prime}$ for all $s \in[a, b]$ (at the points $a$ and $b$, there exist $x^{\prime}(a+0)$ and $x^{\prime}(b-0)$ ) and the derivative is bounded on $[a, b]$, then the function $x$ is absolutely continuous on $[a, b]$. Therefore, the condition below implies conditions $(i i i)$ and $(i v)$ :

$\left(i i i^{\prime}\right)$ the derivative $x^{\prime}$ exists on any interval $[a, b]$ except for a finite number of (discontinuity and break) points and is bounded on $(-\infty,+\infty)$ (we assume that $x^{\prime}\left(s_{i}-0\right)$ and $x^{\prime}\left(s_{i}+0\right), i=1,2, \ldots$, exist and are bounded by the same constant).

The set of functions satisfying conditions $(i),(i i)$, and $\left(i i i^{\prime}\right)$ is contained in $M V$. Moreover, all these conditions have a simple geometric interpretation.

All the results of this paper are also valid on a wider set. All the statements are valid if we replace conditions $(i)-(i v)$ by $(i i)$ and the following condition, which is also necessary:

$\left(i^{\prime}\right)$ the function defined on $(-\infty,+\infty)$ has at most countably many discontinuities of the first kind at points $s_{i}$, and decomposition (1.1) is valid, where the function $x_{0}$ is absolutely continuous on any interval $[a, b]$.

It is clear that the set of functions satisfying $(i i)$ and $\left(i^{\prime}\right)$ contains $M V$ and is wider than $M V$.

A fault of condition $\left(i^{\prime}\right)$ is that it contains the function $x_{0}$ but not the original function $x$. In addition, this condition is not illustrative. Therefore, we prefer to use conditions $(i)-(i v)$.

We have not imposed any constraints on the values of jumps $\Delta_{i}$ of the function $x$. Assume that the function $x$ has $l$ discontinuities such that the absolute values of the jumps $\Delta_{i}, i=1,2, \ldots, l$, are "large" and the remaining discontinuities have "small" values of jumps. Without loss of generality, assume that the first $l$ discontinuities $s_{i}$ are numbered in ascending order; i.e., $s_{i}<s_{k}$ for $i<k$. Under these conditions, for a function $x \in M V$, we will construct in Section 3 methods for localizing (approximating) the points $s_{i}, i=1,2, \ldots, l$, for the approximately given function.

The condition on the values of jumps involves an averaging function $\phi \in W_{1}^{1}(-\infty,+\infty)$. Additional requirements for this function are given in the next section. We set $\phi_{\lambda}(s)=\phi(s / \lambda)$ (in what follows, $\lambda>0$ is a regularization parameter). We are interested in the auxiliary function

$$
x_{\lambda}(s)=\int_{-\infty}^{+\infty} x(t)\left(\phi_{\lambda}(s-t)\right)_{s}^{\prime} d t
$$

for which the main decomposition will be obtained.

Lemma 2. Assume that $x \in M V$ and $\phi \in W_{1}^{1}(-\infty,+\infty)$. Then, for any $\lambda>0$, there exists a continuous function $x_{\lambda}$ given by formula (1.2) such that the following representation holds:

$$
x_{\lambda}(s)=\sum_{i=1}^{\infty} \Delta_{i} \phi_{\lambda}\left(s-s_{i}\right)+\int_{-\infty}^{\infty} \phi_{\lambda}(s-t) x_{0}^{\prime}(t) d t .
$$


Proof. Note that, since the function $\phi$ (and, consequently, $\phi_{\lambda}(s)$ ) belongs to the Sobolev space $W_{1}^{1}[a, b]$, this function is absolutely continuous on any interval $[a, b][18$, Ch. 9, Sect. 8]. The absolute continuity of the functions $\phi_{\lambda}$ and $x_{0}$ implies (see, for example, $[18$, Ch. 9, Sect. 7]) the possibility of integration by parts in the following integral:

$$
\begin{gathered}
\int_{a}^{b} x(t) d \phi_{\lambda}(s-t)=-\left.x(t) \phi_{\lambda}(s-t)\right|_{a} ^{b}+\int_{a}^{b} \phi_{\lambda}(s-t) d x(t) \\
=-\left.x(t) \phi_{\lambda}(s-t)\right|_{a} ^{b}+\int_{a}^{b} \phi_{\lambda}(s-t) x_{0}^{\prime}(t) d t+\sum_{i=1}^{\infty} \Delta_{i} \phi_{\lambda}\left(s-s_{i}\right) .
\end{gathered}
$$

Since $\phi \in W_{1}^{1}(-\infty,+\infty)$, we have $\lim _{t \rightarrow \pm \infty} \phi(t)=0$. The boundedness of the function $x$ implies that we can pass to the limit as $a \rightarrow-\infty$ and $b \rightarrow+\infty$ in this equality. Hence, all the integrals exist and, since the derivatives $x^{\prime}$ and $x_{0}^{\prime}$ coincide almost everywhere, we have the required equality. The lemma is proved.

Let us formulate conditions on the values of the first $l$ discontinuities and their separability from the remaining discontinuities:

(1) a number $\Delta^{\text {min }}>0$ is given such that $\min \left\{\left|\Delta_{i}\right|: i=1,2, \ldots, l\right\} \geq \Delta^{\min }$;

(2) a number $\lambda_{0}>0$ is given such that the following inequality is valid for all $0<\lambda \leq \lambda_{0}$ :

$$
\mathfrak{d}_{l}=\sup _{s} \sum_{i=l+1}^{\infty}\left|\Delta_{i} \phi_{\lambda}\left(s-s_{i}\right)\right| \leq \gamma \Delta^{\mathrm{min}}, \quad 0 \leq \gamma<1 / 2 .
$$

Let us agree that the function $x$ has at least $l$ discontinuities; if their number is exactly $l$, then we assume that $\mathfrak{d}_{l}=0$. Note that condition (1) was used in [12-17]. As for the condition of "smallness" of the remaining discontinuities, it is clear that condition (2) is too complicated and nontransparent. In addition, it contains the function $\phi_{\lambda}$, which specifies the regularization method and is not connected with the exact function $x$. Therefore, it is desirable to have simpler sufficient conditions. A lot of variants are possible; we consider only three of them. The first one is the simplest condition of the smallness of the remaining discontinuities "in total":

(2a) $\quad \sum_{i=l+1}^{\infty}\left|\Delta_{i}\right| \leq \gamma \Delta^{\text {min }}$, where $0 \leq \gamma<1 / 2$.

Under the assumption that the function $\phi$ satisfies the condition $\sup _{t \in[-1,1]}|\phi(t)|=1$, condition (2a) implies condition (2) for any $\lambda>0$. By contrast with the preceding condition, let us introduce a weaker condition on the values $\left|\Delta_{i}\right|, i=l+1, l+2, \ldots$ :

(2b) $\sup \left\{\left|\Delta_{i}\right|: i=l+1, l+2, \ldots\right\} \leq \gamma \Delta^{\text {min }}$, where $0 \leq \gamma<1 / 2$.

This condition also implies condition (2) under additional assumptions on the function $\phi$ and the location of discontinuities.

Lemma 3. Assume that $\phi(t)=0$ for $t \notin[-1,1]$ and $\sup _{t \in[-1,1]}|\phi(t)|=1$. Then, if there exists $h>0$ such that

$$
t \in[-1,1]
$$

$$
\inf _{k, i \geq 1, k \neq i}\left|s_{k}-s_{i}\right| \geq h,
$$

then condition (2b) implies condition (2) for $\lambda_{0}=h / 2$. 
Proof. We have $\phi(t)=0$ for $t \notin[-1,1]$; hence, the inequality

$$
\mathfrak{d}_{l}=\sup _{i \geq l+1}\left|\Delta_{i}\right| \sup _{s} \sum_{i=l+1}^{\infty}\left|\phi_{\lambda}\left(s-s_{i}\right)\right| \leq \sup _{i \geq l+1}\left|\Delta_{i}\right|
$$

is valid for $0<\lambda \leq \lambda_{0}=h / 2$. By condition (2b), we obtain the required inequality $\mathfrak{d}_{l} \leq \gamma \Delta^{\text {min }}$, where $0 \leq \gamma<1 / 2$. The lemma is proved.

The third variant with exactly $l$ discontinuities was considered earlier in [12-17]. In this variant, condition (2) holds for $\gamma=0$ automatically; therefore, only condition (1) was used in these papers.

\section{LOCALIZATION METHOD AND ITS INVESTIGATION}

Let us first formulate the problem of localization of discontinuities of the first kind for a function from the space $M V$. Let us agree that, everywhere in what follows, $\|\cdot\|_{L_{1}}$ and $\|\cdot\|_{L_{2}}$ mean $\|\cdot\|_{L_{1}(-\infty,+\infty)}$ and $\|\cdot\|_{L_{2}(-\infty,+\infty)}$, respectively.

The problem of localization of discontinuities in $\boldsymbol{M V}$. Assume that $x \in M V$ and the first $l$ discontinuities belong to a known interval $(-d, d), d>0$. For a known function $x^{\delta}$ such that $\left\|x-x^{\delta}\right\|_{L_{2}} \leq \delta$ and the error level $\delta$, it is required to find the number $l$ and approximate the positions of the first $l$ discontinuities of the first kind $\left\{s_{i}\right\}_{1}^{l}$ of the function $x$.

In practice, sometimes, it is also necessary to approximate the values of jumps $\left\{\Delta_{i}\right\}_{1}^{l}$. This problem is simpler; we do not construct such algorithms in the present paper (see, e.g., [13-15]).

Looking ahead, note that, for any function $x \in M V$, the localization method constructed below converges under additional conditions (1) and (2), and the number of "large" discontinuities can be found for all $\delta \leq \delta_{0}(x)$. Unfortunately, the value $\delta_{0}(x)$ can be arbitrarily small. Let us introduce additional conditions for the function $x$ and obtain uniform estimates for the accuracy of localization of discontinuities, all constants in which are independent of the function $x$. These uniform estimates are similar to the uniform estimates in the classical theory of ill-posed problems on classes of well-posedness [2, Ch. 4]. Denote supremum almost everywhere by esssup.

Assume that additional a priori information about the exact function $x$ is known:

(3) a number $r>0$ is given such that $\operatorname{esssup}_{s}\left|x^{\prime}(s)\right| \leq r$;

(4) $0<l \leq L$ and $\max \left\{\left|\Delta_{i}\right|: i=1,2, \ldots, l\right\} \leq \Delta^{\max }$ for positive numbers $L$ and $\Delta^{\max }$.

In what follows, without loss of generality, we assume that the number $r$ in condition (3) is equal to one. Denote by $\mathfrak{M}_{M V}$ the set of exact functions $x \in M V$ satisfying conditions (1)-(4).

Denote by $\Phi$ the set of averaging functions $\phi(s)$ satisfying the following conditions:

(a) $\phi \in W_{1}^{1}(-\infty,+\infty)$ and $\left\|\phi^{\prime}\right\|_{L_{2}}<\infty$;

(b) $\sup \{|\phi(t)|: t \in[-1,1]\}=\phi(0)=1$;

(c) $|\phi(t)| \leq C /|t|$ for $t \notin[-1,1]$, where $C$ is a constant.

Denote by $\Phi F$ the set of functions $\phi(s)$ satisfying conditions $(a)$ and $(b)$ and the condition

$\left(c^{\prime}\right) \phi(t)=0$ for $t \notin[-1,1]$.

It is clear that $\Phi F \subset \Phi$. Consider examples of averaging functions:

$$
\begin{gathered}
\phi_{1}[\sigma](t)=\exp \left(-\frac{t^{2}}{2 \sigma}\right), \quad \sigma>0 \text { is a fixed parameter, } \\
\phi_{2}(t)=\left\{\begin{array}{ll}
\cos ^{2}\left(\frac{\pi t}{2}\right), & t \in[-1,1], \\
0, & t \notin[-1,1],
\end{array} \quad \phi_{3}(t)= \begin{cases}1-|t|, & t \in[-1,1], \\
0, & t \notin[-1,1] .\end{cases} \right.
\end{gathered}
$$


We have $\phi_{1}[\sigma] \in \Phi$ and $\phi_{2}, \phi_{3} \in \Phi F$.

To localize singularities, we construct and investigate the auxiliary function

$$
x_{\lambda}^{\delta}(s)=\int_{-\infty}^{+\infty} x^{\delta}(t)\left(\phi_{\lambda}(s-t)\right)_{s}^{\prime} d t
$$

Recall that $\gamma$ and $\lambda_{0}$ are the constants from condition (2).

Lemma 4. Assume that $x \in \mathfrak{M}_{M V}$ and $\phi \in \Phi$. Then, for any $0<\lambda \leq \lambda_{0}$, there exists a continuous function $x_{\lambda}^{\delta}$ given by formula (2.1) such that the following representation holds:

$$
x_{\lambda}^{\delta}(s)=\sum_{i=1}^{l} \Delta_{i} \phi_{\lambda}\left(s-s_{i}\right)+\alpha_{\lambda}(s)+\alpha_{\lambda}^{l}(s)+\Delta x_{\lambda}^{\delta}(s),
$$

where

$$
\sup _{s}\left|\alpha_{\lambda}(s)\right| \leq A_{0} \lambda, \quad \sup _{s}\left|\alpha_{\lambda}^{l}(s)\right| \leq \gamma \Delta^{\min }, \quad \sup _{s}\left|\Delta x_{\lambda}^{\delta}(s)\right| \leq A_{1} \lambda^{-1 / 2} \delta,
$$

$A_{0}=\|\phi\|_{L_{1}}$, and $A_{1}=\left\|\phi^{\prime}\right\|_{L_{2}}$.

Proof. Write the function $x_{\lambda}^{\delta}(s)$ as the sum of two functions:

$$
\begin{gathered}
x_{\lambda}^{\delta}(s)=x_{\lambda}(s)+\Delta x_{\lambda}^{\delta}(s), \quad \text { where } \quad x_{\lambda}(s)=\int_{-\infty}^{+\infty} x(t)\left(\phi_{\lambda}(s-t)\right)_{s}^{\prime} d t \\
\Delta x_{\lambda}^{\delta}(s)=\int_{-\infty}^{+\infty}\left(x^{\delta}(t)-x(t)\right)\left(\phi_{\lambda}(s-t)\right)_{s}^{\prime} d t .
\end{gathered}
$$

Using the Hölder inequality for integral (2.3), we estimate the absolute value of the function $\Delta x_{\lambda}^{\delta}$ :

$$
\left|\Delta x_{\lambda}^{\delta}(s)\right| \leq\left\|x^{\delta}-x\right\|_{L_{2}}\left\|\left(\phi_{\lambda}(s-t)\right)_{s}^{\prime}\right\|_{L_{2}} .
$$

Since $\left\|\left(\phi_{\lambda}(s-t)\right)_{s}^{\prime}\right\|_{L_{2}} \leq \lambda^{-1 / 2}\left\|\phi^{\prime}\right\|_{L_{2}}$, we obtain the required estimate.

Consider the function $x_{\lambda}$. In Lemma 2 , it is shown that

$$
x_{\lambda}(s)=\sum_{i=1}^{\infty} \Delta_{i} \phi_{\lambda}\left(s-s_{i}\right)+\int_{-\infty}^{\infty} \phi_{\lambda}(s-t) x^{\prime}(t) d t
$$

Define

$$
\alpha_{\lambda}^{l}(s)=\sum_{i=l+1}^{\infty} \Delta_{i} \phi_{\lambda}\left(s-s_{i}\right), \quad \alpha_{\lambda}(s)=\int_{-\infty}^{\infty} \phi_{\lambda}(s-t) x^{\prime}(t) d t .
$$

Using condition (3) for the function $x$, we obtain the estimate

$$
\left|\alpha_{\lambda}(s)\right| \leq \int_{-\infty}^{+\infty}\left|\phi_{\lambda}(s-t)\right| d t=\lambda \int_{-\infty}^{+\infty}|\phi(u)| d u \leq\|\phi\|_{L_{1}} \lambda .
$$

The required estimate for $\alpha_{\lambda}^{l}(s)$ follows from condition (2). The lemma is proved.

PROCEEDINGS OF THE STEKLOV INSTITUTE OF MATHEMATICS $\quad$ Vol. $280 \quad$ Suppl. $1 \quad 2013$ 
The method of localization of discontinuities of the first kind consists in the investigation of the function $\left|x_{\lambda}^{\delta}(s)\right|$ and is similar to the method presented in [17]. We approximate discontinuity points in two steps. At the first step, we define a number $m$ (it will be proved later that $m=l$ ) and find disjoint intervals $\left[a_{i}, b_{i}\right], i=1,2, \ldots, m$, that contain the points $s_{i}$. At the second step, we find approximations $s_{i}^{\delta} \in\left[a_{i}, b_{i}\right]$ to the points $s_{i}$ and estimate the accuracy of approximation. Let us introduce the parameter $P=\Delta^{\text {min }} / 2$. The method also uses parameters $\lambda$ and $h$ defined below.

Method $\Pi$. For the function $x^{\delta}$, calculate an auxiliary function $x_{\lambda}^{\delta}$ by formula (2.1). Set $\tilde{s}:=-d-1$ and $m:=0$.

Step of the method. If the equation $\left|x_{\lambda}^{\delta}(s)\right|-P=0$ has no roots on the interval $[\tilde{s}, d]$, then the process is complete; otherwise, denote by $\bar{s}$ the leftmost root and set $m:=m+1, a_{m}:=\bar{s}$, $b_{m}:=\bar{s}+h / 3$, and $\tilde{s}:=b_{m}+h / 3$. Repeat the step.

Method $\Pi \boldsymbol{\Pi}$. Choose $s_{i}^{\delta}$ as the bisecting point of the interval $\left[a_{i}, b_{i}\right], i=1,2, \ldots, m$.

Recall that $\Delta^{\min }, \gamma, \lambda_{0}, L$, and $\Delta^{\max }$ are the constants from the conditions for the function $x$; $C$ is the constant from the conditions for $\phi ; A_{0}=\|\phi\|_{L_{1}}$; and $A_{1}=\left\|\phi^{\prime}\right\|_{L_{2}}$. To formulate theorems, we need the following numbers:

$$
\begin{gathered}
D=\left(\frac{8 A_{1}}{(a-2 \gamma) \Delta^{\min }}\right)^{2}, \quad \delta_{0}=\frac{1}{D^{1 / 2}} \min \left\{\left(\frac{\Delta^{\min }(a-2 \gamma)}{8 A_{0}}\right)^{1 / 2}, \quad \lambda_{0}^{1 / 2}\right\}, \\
H=\frac{8 L \Delta^{\max } \tilde{C}}{\Delta^{\min }(a-2 \gamma)}, \quad \text { where } \tilde{C}=\max \left\{C, \frac{\Delta^{\min }(a-2 \gamma)}{8 L \Delta^{\max }}\right\} .
\end{gathered}
$$

The following three theorems contain results on the localization of discontinuities for different sets of averaging functions, i.e., for different groups of localization methods.

Theorem 1. Assume that $x \in \mathfrak{M}_{M V} ; \phi \in \Phi$; the constants $D, \delta_{0}$, and $H$ are given by equalities (2.4) and (2.5) for $a=1 ; \delta \leq \delta_{0}$; the parameters are connected by the equality $\lambda(\delta)=D \delta^{2} ;$ and $\min _{1 \leq i, k \leq l, i \neq k}\left|s_{i}-s_{k}\right| \geq h(\delta)$ for $h(\delta)=3 H \lambda(\delta)$. Then, $m=l$ and the estimate $\left|s_{i}^{\delta}-s_{i}\right| \leq(H D / 2) \delta^{2}$ is valid for method $\Pi-\Pi F$.

Proof. Consider the function $x_{\lambda}^{\delta}(s)$ in a neighborhood of a point $s_{i}$ such that $\left|s-s_{i}\right| \leq H \lambda$, $i=1,2, \ldots, l$. Using representation $(2.2)$, we obtain

$$
x_{\lambda}^{\delta}(s)=\Delta_{i} \phi_{\lambda}\left(s-s_{i}\right)+\alpha_{\lambda}(s)+\alpha_{\lambda}^{l}(s)+\Delta x_{\lambda}^{\delta}(s)+\sum_{k=1(k \neq i)}^{l} \Delta_{k} \phi_{\lambda}\left(s-s_{k}\right) .
$$

In view of condition $(c)$ for the function $\phi$, for all $s$ such that $\left|s-s_{k}\right| \geq H \lambda$, we have the following estimate for the last additive term:

$$
\left|\sum_{k=1(k \neq i)}^{l} \Delta_{k} \phi_{\lambda}\left(s-s_{k}\right)\right| \leq \frac{(L-1) C \Delta^{\max }}{H} .
$$

Note that $\lambda \leq \lambda_{0}$ for $\delta \leq \delta_{0}$ and $\lambda=\lambda(\delta)$. Then, for $i=1,2, \ldots, l$, using the estimates from Lemma 4, we get

$$
\sup _{\left|s-s_{i}\right| \leq H \lambda}\left|\alpha_{\lambda}(s)+\alpha_{\lambda}^{l}(s)+\Delta x_{\lambda}^{\delta}(s)+\sum_{k=1(k \neq i)}^{l} \Delta_{k} \phi_{\lambda}\left(s-s_{k}\right)\right|
$$




$$
\leq A_{0} \lambda+A_{1} \delta \lambda^{-1 / 2}+\frac{(L-1) C \Delta^{\max }}{H}+\gamma \Delta^{\min }
$$

By property $(b)$ of the function $\phi$, for these parameters and $i=1,2, \ldots, l$, we obtain the estimate

$$
\sup _{\left|s-s_{i}\right| \leq H \lambda}\left|x_{\lambda}^{\delta}(s)\right| \geq\left|x_{\lambda}^{\delta}\left(s_{i}\right)\right| \geq \Delta^{\min }-\frac{3(1-2 \gamma)}{8} \Delta^{\min }-\gamma \Delta^{\min }>\frac{\Delta^{\min }}{2}=P,
$$

since $0 \leq \gamma<1 / 2$. Outside the set $Q=\bigcup_{i=1}^{l}\left\{s:\left|s-s_{i}\right| \leq H \lambda\right\}$, the function $\left|x_{\lambda}^{\delta}(s)\right|$ is estimated from above as follows:

$$
\left|x_{\lambda}^{\delta}(s)\right| \leq A_{0} \lambda+A_{1} \delta \lambda^{-1 / 2}+\frac{L \Delta^{\max } C}{H}+\gamma \Delta^{\min } \leq \frac{3(1-2 \gamma)}{8} \Delta^{\min }+\gamma \Delta^{\min }<\frac{\Delta^{\min }}{2}=P .
$$

To simplify the presentation, we carry out the further proof for $l=2$; i.e., we perform two steps of method $\Pi$ and, at each of the steps, find an interval containing one point $s_{i}$. The proof for arbitrary $l$ is similar: $l$ steps of method $\Pi$ must be performed. Recall that we consider the problem on the set of functions $x_{\lambda}^{\delta}(s)$ for which $s_{2}-s_{1} \geq h(\delta)$.

In view of estimates (2.8) and (2.9) and the continuity of the function $\left|x_{\lambda}^{\delta}\right|$, in method $\Pi$, there exists a point $\bar{s}$ such that $\left|x_{\lambda}^{\delta}(\bar{s})\right|=P$. Since $s_{1}<s_{2}$, we have $\left|\bar{s}-s_{1}\right| \leq H \lambda$. It follows from (2.8) that $\left|x_{\lambda}^{\delta}\left(s_{1}\right)\right|>P$. Hence, $s_{1}>\bar{s}$. Consequently, $s_{1}$ belongs to the interval $[\bar{s}, \bar{s}+H \lambda]=\left[a_{1}, b_{1}\right]$.

Consider the interval $\left[b_{1}+H \lambda, d\right]$. It is clear that this interval contains no points $s$ such that $\left|s-s_{1}\right| \leq H \lambda$. On the other hand, since $s_{2}-s_{1} \geq 3 H \lambda$, we have $b_{1}+H \lambda<s_{2}-H \lambda$. Consequently, the leftmost root $\bar{s}$ of the equation $\left|x_{\lambda}^{\delta}(s)\right|-P=0$ on the interval $\left[b_{1}+H \lambda, d\right]$ is strictly less than $s_{2}$. By (2.8), the point $s_{2}$ belongs to the interval $[\bar{s}, \bar{s}+H \lambda]=\left[a_{2}, b_{2}\right]$.

The intervals $\left[a_{1}, b_{1}\right]$ and $\left[a_{2}, b_{2}\right]$ are disjoint, since $a_{2}-b_{1} \geq H \lambda>0$.

Consider the interval $\left[b_{2}+H \lambda, d\right]$. Clearly, it contains no points from the set $Q$. Hence, the equation $\left|x_{\lambda}^{\delta}(s)\right|-P=0$ has no roots on this interval. Thus, $m=2$ and the process is complete.

Since $\left|s-s_{i}\right| \leq H \lambda$ for all points $s \in\left[a_{i}, b_{i}\right], i=1,2$, we have $\left|s_{i}^{\delta}-s_{i}\right| \leq(H / 2) \lambda$ for the bisecting points of the intervals. The theorem is proved.

Consider the case when the auxiliary function $x_{\lambda}^{\delta}$ is constructed for an averaging function $\phi$ from the set $\Phi F$. In this case, the last additive term in decomposition (2.6) is absent; this improves the estimates both in accuracy and in separability (we can take a better function $h(\delta)$ in the theorem). In addition, all the constants do not contain the values $\Delta^{\max }$ and $L$; this gives an essentially better result in the case when $\Delta^{\max } \gg \Delta^{\min }$ and (or) $L$ is a large number.

Theorem 2. Assume that a function $x \in M V$ satisfies conditions (1), (2), and (3); $\phi \in \Phi F$; constants $D$ and $\delta_{0}$ are given by equalities (2.4) for $a=1 ; \delta \leq 2 \delta_{0}$; the parameters are connected by the equality $\lambda(\delta)=(D / 4) \delta^{2} ;$ and $\min _{1 \leq i, k \leq l, i \neq k}\left|s_{i}-s_{k}\right| \geq h(\delta)$ for $h(\delta)=3 \lambda(\delta)$. Then, $m=l$ and the estimate $\left|s_{i}^{\delta}-s_{i}\right| \leq(D / 8) \delta^{2}$ is valid for method $\Pi-\Pi F$.

The proof of this theorem is completely similar to the proof of Theorem 1.

Consider the case when the auxiliary function $x_{\lambda}^{\delta}$ is constructed for a function $\phi \in \Phi$ with the additional condition

(d) $\sup _{t \in[-1,1]}|\phi(t)|-\sup _{t \notin[-1,1]}|\phi(t)|=a>0$.

It is clear that, for a function $\phi \in \Phi F$, this condition holds for $a=1$. In this case, we can obtain a better estimate for the accuracy of localizing the positions of discontinuities in comparison with Theorem 1. To this end, we should use the following method instead of method $\Pi F$. 
Method П1. Find a point $s_{i}^{\delta}$ of maximum of the function $\left|x_{\lambda}^{\delta}(s)\right|$ on each interval $\left[a_{i}, b_{i}\right]$, $i=1,2, \ldots, m$. If there are several such points, take the leftmost of them.

Theorem 3. Assume that condition $(d)$ holds for a function $\phi \in \Phi ; x \in \mathfrak{M}_{M V} ; \gamma \leq a / 2$; constants $D, \delta_{0}$, and $H$ are given by equalities (2.4) and (2.5), where the parameter a is taken from condition $(d) ; \delta \leq \delta_{0}$; the parameters are connected by the equality $\lambda(\delta)=D \delta^{2}$; and $\min _{1 \leq i, k \leq l, i \neq k}\left|s_{i}-s_{k}\right| \geq h(\delta)$ for $h(\delta)=3 H \lambda(\delta)$. Then, $m=l$ and the estimate $\left|s_{i}^{\delta}-s_{i}\right| \leq D \delta^{2}$ is valid for method $\Pi-\Pi 1$.

Proof. The proof of the fact that, in this case, method $\Pi$ finds the number $l$ and disjoint intervals that contain the points $s_{i}$ is similar to the proof in Theorem 1 . Let us show the validity of the estimate for the approximation $s_{i}^{\delta}$ constructed by method $\Pi 1$.

Let $s_{i}^{\delta}$ be the maximum point for the absolute value of the function $x_{\lambda}^{\delta}(s)$ on the interval $\left[a_{i}, b_{i}\right], i=1,2$. Using conditions $(b)$ and $(d)$ for the function $\phi$, we obtain

$$
\sup _{\left|s-s_{i}\right|>\lambda}\left|\phi_{\lambda}\left(s-s_{i}\right)\right|=1-a \geq 0 .
$$

Using decomposition (2.6) and estimate (2.7), for $i=1,2$, we obtain

$$
\left|x_{\lambda}^{\delta}\left(s_{i}^{\delta}\right)\right|>\Delta_{i}-\frac{a \Delta^{\min }}{2} \geq \frac{(2-a) \Delta_{i}}{2}, \quad \sup _{\left|s-s_{i}\right|>\lambda}\left|x_{\lambda}^{\delta}(s)\right|<\Delta_{i}(1-a)+\frac{a \Delta^{\min }}{2} \geq \frac{(2-a) \Delta_{i}}{2} .
$$

Thus, $\left|s_{i}^{\delta}-s_{i}\right| \leq \lambda, i=1,2$. The theorem is proved.

Consider the case when the function $x \in M V$ has exactly $l$ discontinuities of the first kind. In this case, condition (2) holds for $\gamma=0$ automatically. For this case, we combine the results similar to Theorems 1-3 into one theorem. We introduce the corresponding constants

$$
\begin{gathered}
D=\left(\frac{8 A_{1}}{a \Delta^{\min }}\right)^{2}, \quad \delta_{0}=\left(\frac{a \Delta^{\min }}{4 D A_{0}}\right)^{1 / 2}, \\
H=\frac{8 L \Delta^{\max } \tilde{C}}{a \Delta^{\min }}, \quad \text { where } \quad \tilde{C}=\max \left\{C, \frac{a \Delta^{\min }}{8 L \Delta^{\max }}\right\} .
\end{gathered}
$$

Theorem 4. Let a function $x \in M V$ have exactly $l$ discontinuities of the first kind and satisfy conditions (1) and (3).

(A) Assume that $\phi \in \Phi$; the function $x$ additionally satisfies condition (4); constants $D, \delta_{0}$, and $H$ are given by equalities (2.10) and (2.11) for $a=1 ; \delta \leq \delta_{0}$; the parameters are connected by the equality $\lambda(\delta)=D \delta^{2} ;$ and $\min _{1 \leq i, k \leq l, i \neq k}\left|s_{i}-s_{k}\right| \geq h(\delta)$ for $h(\delta)=3 H \lambda(\delta)$. Then, $m=l$ and the estimate $\left|s_{i}^{\delta}-s_{i}\right| \leq(H D / 2) \delta^{2}$ is valid for method $\Pi-\Pi F$.

(B) Assume that $\phi \in \Phi F$; constants $D$ and $\delta_{0}$ are given by equalities (2.10) for $a=1 ; \delta \leq 2 \delta_{0}$; the parameters are connected by the equality $\lambda(\delta)=(D / 4) \delta^{2} ;$ and $\min _{1 \leq i, k \leq l, i \neq k}\left|s_{i}-s_{k}\right| \geq h(\delta)$ for $h(\delta)=3 \lambda(\delta)$. Then, $m=l$ and the estimate $\left|s_{i}^{\delta}-s_{i}\right| \leq(D / 8) \delta^{2}$ is valid for method $\Pi-\Pi F$.

(C) Assume that a function $\phi \in \Phi$ satisfies condition $(d)$; the function $x$ additionally satisfies condition (4); constants $D, \delta_{0}$, and $H$ are given by equalities $(2.10)$ and $(2.11) ; \delta \leq \delta_{0}$; the parameters are connected by the equality $\lambda(\delta)=D \delta^{2} ;$ and $\min _{1 \leq i, k \leq l, i \neq k}\left|s_{i}-s_{k}\right| \geq h(\delta)$ for $h(\delta)=3 H \lambda(\delta)$. Then, $m=l$ and the estimate $\left|s_{i}^{\delta}-s_{i}\right| \leq D \delta^{2}$ is valid for method $\Pi-\Pi 1$.

This theorem is a consequence of Theorems 1-3. 


\section{THE OPTIMALITY OF THE LOCALIZATION METHODS \\ ON CLASSES OF FUNCTIONS WITH DISCONTINUITIES AND EXAMPLES OF SPECIFIC LOCALIZATION METHODS}

To establish the (order) optimality of a specific method, it is necessary to estimate the attainable accuracy of approximation of the positions of singularities from below. Effective estimates of this kind are also of independent interest.

Let us introduce a condition for a function with singularities:

(5) a positive number $\hat{h}$ is given such that $\min _{1 \leq i, k \leq l, i \neq k}\left|s_{i}-s_{k}\right| \geq \hat{h}$.

Denote by $\mathfrak{M}_{M V}^{\prime}$ the class of functions from $\mathfrak{M}_{M V}$ satisfying condition (5).

Definition 1. A method that, for a function $x^{\delta}$ and error level $\delta$, finds the value $l$ and approximations $s_{i}^{\delta}, i=1,2, \ldots, l$, to positions of singularities $s_{i}$ of the function $x$ is called a localization method.

For an arbitrary localization method $\tilde{\Pi}$ on the class of functions $\mathfrak{M}_{M V}^{\prime}$, we introduce the notion of optimality and order optimality and define a method that is optimal (order optimal) in accuracy.

Definition 2. For a method $\tilde{\Pi}$, the accuracy of recovery of singularities on the class $\mathfrak{M}_{M V}^{\prime}$ is defined by the value

$$
\tau\left(\mathfrak{M}_{M V}^{\prime}, \tilde{\Pi}, \delta\right) \equiv \sup _{x \in \mathfrak{M}_{M V}^{\prime}} \sup _{\left\|x-x^{\delta}\right\|_{C} \leq \delta} \sup _{1 \leq i \leq l}\left|s_{i}-s_{i}^{\delta}\right|
$$

The value $\hat{\tau}\left(\mathfrak{M}_{M V}^{\prime}, \delta\right)=\min _{\tilde{\Pi}} \tau\left(\mathfrak{M}_{M V}^{\prime}, \tilde{\Pi}, \delta\right)$ is called the optimal accuracy of localization of singularities on the class $\mathfrak{M}_{M V}^{\prime}$ (the minimum is taken over all methods of localization of discontinuities). A method $\tilde{\Pi}$ is called optimal (order optimal) on the class $\mathfrak{M}_{M V}^{\prime}$ if $\hat{\tau}\left(\mathfrak{M}_{M V}^{\prime}, \delta\right)=\tau\left(\mathfrak{M}_{M V}^{\prime}, \tilde{\Pi}, \delta\right)$ (there exists a constant $R>1$ such that $\tau\left(\mathfrak{M}_{M V}^{\prime}, \tilde{\Pi}, \delta\right) \leq R \hat{\tau}\left(\mathfrak{M}_{M V}^{\prime}, \delta\right)$ ).

In addition to the accuracy of approximation, it is interesting to estimate from below the separability threshold, which is another characteristic of localization methods.

Definition 3. The smallest function $\bar{h}\left(\delta, \tilde{\Pi}, \mathfrak{M}_{M V}\right)$ that can be taken in the condition $\min _{1 \leq i, k \leq l, i \neq k}\left|s_{i}-s_{k}\right| \geq \bar{h}\left(\delta, \tilde{\Pi}, \mathfrak{M}_{M V}\right)$ so that method $\tilde{\Pi}$ still localizes singularities is called the separability threshold for method $\tilde{\Pi}$ on the class of functions $\mathfrak{M}_{M V}$; the function $\hat{h}\left(\delta, \mathfrak{M}_{M V}\right)=$ $\min _{\tilde{\Pi}} \bar{h}\left(\delta, \tilde{\Pi}, \mathfrak{M}_{M V}\right)$, where the minimum is taken over all localization methods $\tilde{\Pi}$, is called the separability threshold for the problem on the class of functions $\mathfrak{M}_{M V}$. A method $\tilde{\Pi}$ is called P-optimal (order P-optimal) on the class $\mathfrak{M}_{M V}$ if $\hat{h}\left(\mathfrak{M}_{M V}, \delta\right)=\bar{h}\left(\mathfrak{M}_{M V}, \tilde{\Pi}, \delta\right)$ (there exists a constant $R>1$ such that $\left.\bar{h}\left(\mathfrak{M}_{M V}, \tilde{\Pi}, \delta\right) \leq R \hat{h}\left(\mathfrak{M}_{M V}, \delta\right)\right)$.

Corollary 1. In Theorems 1-4, the following upper estimates are obtained for the separability threshold of the methods considered in these theorems: $\bar{h}\left(\delta, \tilde{\Pi}, \mathfrak{M}_{M V}\right) \leq h(\delta)$.

In [19], lower estimates are proved for the optimal accuracy and separability threshold in the problem under consideration for a noisy function in $L_{p}(-\infty,+\infty)$. For the problem considered in the present paper, for $p=2$,

$$
\hat{\tau}\left(\mathfrak{M}_{M V}, \delta\right) \geq\left(\frac{\delta}{\Delta^{\min }}\right)^{2}, \quad \hat{h}\left(\mathfrak{M}_{M V}^{\prime}, \delta\right) \geq\left(\frac{\delta}{\Delta^{\min }}\right)^{2}
$$


As noted above, each of Theorems 1-3 allows one to obtain specific methods in particular situations. Let us exemplify these theorems and construct several specific methods of localization of discontinuities of a noisy function from the space $M V$ using specific averaging functions under more specific conditions on the exact function.

We take $\phi_{1}[\sigma]$ for the averaging function (see examples in Section 2). Since $\phi_{1}[\sigma] \in \Phi$, we can require condition (2a) instead of condition (2) for the function $x$. Since $\left\|\phi_{1}\right\|_{L_{1}}=\sqrt{2 \pi \sigma}$ and $\left\|\phi_{1}^{\prime}\right\|_{L_{2}}=\sqrt{\pi^{1 / 2} /\left(2 \sigma^{1 / 2}\right)}$, according to $(2.4)$ and (2.5), we obtain the following constants for $a=1$ :

$$
D=\left(\frac{\pi}{\sigma}\right)^{1 / 2} \frac{32}{\left((1-2 \gamma) \Delta^{\min }\right)^{2}}, \quad \delta_{0}=\left(\frac{\Delta^{\min }(1-2 \gamma)}{2 \sqrt{2 \pi \sigma} D}\right)^{1 / 2}, \quad H=\frac{8 L \Delta^{\max } \exp (-1 /(2 \sigma))}{\Delta^{\min }(1-2 \gamma)}
$$

Following method $\Pi$, we calculate the auxiliary function $x_{\lambda}^{\delta}$ by formula (2.1). To localize discontinuities, we use method $\Pi-\Pi F$ with the parameters $\lambda(\delta)=D \delta^{2}$ and $h(\delta)=3 H \lambda(\delta)$. The following statement is a consequence of Theorem 1.

Corollary 2. Assume that a function $x \in M V$ satisfies conditions (1), (2a), (3), and (4); $\delta \leq \delta_{0}$; the parameters are connected by the equality $\lambda=\lambda(\delta) ;$ and $\min _{1 \leq i, k \leq l, i \neq k}\left|s_{i}-s_{k}\right| \geq h(\delta)$. Then, $m=l$ and the estimate $\left|s_{i}^{\delta}-s_{i}\right| \leq(H D / 2) \delta^{2}$ is valid for method $\Pi-\Pi F$. This localization method is optimal and order P-optimal on the corresponding classes of functions with discontinuities.

Note that the function $\phi_{1}[\sigma]$ satisfies condition $(d)$ for $a=1-\exp (-1 /(2 \sigma))$. Consequently, if the parameter $\gamma$ from condition (2a) is such that $\gamma \leq a / 2$, then the localization method constructed on the base of the function $\phi_{1}[\sigma]$ allows one to obtain better approximations to the positions of singularities. According to (2.4) and (2.5), we have

$$
D=\left(\frac{\pi}{\sigma}\right)^{1 / 2} \frac{32}{\left((a-2 \gamma) \Delta^{\min }\right)^{2}}, \quad \delta_{0}=\left(\frac{\Delta^{\min }(a-2 \gamma)}{2 \sqrt{2 \pi \sigma} D}\right)^{1 / 2}, \quad H=\frac{8 L \Delta^{\max } \exp (-1 /(2 \sigma))}{\Delta^{\min }(a-2 \gamma)}
$$

To localize discontinuities, we use method $\Pi-\Pi 1$ with the parameters $\lambda(\delta)=D \delta^{2}$ and $h(\delta)=3 H \lambda(\delta)$. The following statement is a consequence of Theorem 3 .

Corollary 3. Assume that a function $x \in M V$ satisfies conditions (1), (2a), (3), and (4); $\gamma \leq a / 2 ; \quad \delta \leq \delta_{0}$; the parameters are connected by the equality $\lambda=\lambda(\delta) ;$ and the inequality $\min _{1 \leq i, k \leq l, i \neq k}\left|s_{i}-s_{k}\right| \geq h(\delta)$ is valid. Then, $m=l$ and the estimate $\left|s_{i}^{\delta}-s_{i}\right| \leq D \delta^{2}$ is valid for method П-П1. This localization method is optimal and order P-optimal on the corresponding classes of functions with discontinuities.

Let us construct a localization method based on the function $\phi_{2}$. Since $\phi_{2} \in \Phi$, we can apply Theorem 1 for this function; i.e., we can obtain estimates similar to the estimates in Corollary 2. In addition, the function $\phi_{2}$ satisfies condition $(d)$ for $a=1$. Hence, we can also obtain estimates based on Theorem 3. However, since $\phi_{2} \in \Phi F$, we can obtain a better approximation to the positions of singularities using method $\Pi F$ instead of method $\Pi 1$. In addition, by Lemma 2, for the function $\phi_{2} \in \Phi F$, condition (2) on the function $x$ follows from weaker condition (2b). Thus, the following statement is a consequence of Theorem 2. Since $\left\|\phi_{2}\right\|_{L_{1}}=1$ and $\left\|\phi_{2}^{\prime}\right\|_{L_{2}}=\pi / 2$, we have

$$
D=\left(\frac{4 \pi}{(1-2 \gamma) \Delta^{\min }}\right)^{2}, \quad \delta_{0}=\left(\frac{\Delta^{\min }(1-2 \gamma)}{2 D}\right)^{1 / 2}, \quad \lambda(\delta)=D \delta^{2}, \quad h(\delta)=3 \lambda(\delta) .
$$


Corollary 4. Assume that a function $x \in M V$ satisfies conditions (1), (2b), and $(3) ; \delta \leq \delta_{0}$; the parameters are connected by the equality $\lambda=\lambda(\delta) ;$ and $\min _{i, k \geq 1, i \neq k}\left|s_{i}-s_{k}\right| \geq h(\delta)$. Then, $m=l$ and the estimate $\left|s_{i}^{\delta}-s_{i}\right| \leq(D / 2) \delta^{2}$ is valid for method $\Pi-\Pi F$. This localization method is optimal and order P-optimal on the corresponding classes of functions with discontinuities.

\section{ACKNOWLEDGMENTS}

This work was supported by the Ural Branch of the Russian Academy of Sciences (project no. 12-P-1-1022) within the Program for Fundamental Research of the Presidium of the Russian Academy of Sciences "Dynamic Systems and Control Theory" and by the Russian Foundation for Basic Research (project no. 09-01-00053).

\section{REFERENCES}

1. A. N. Tikhonov and V. Ya. Arsenin, Solutions of Ill-Posed Problems (Nauka, Moscow, 1974; Wiley, New York, 1977).

2. V. K. Ivanov, V. V. Vasin, and V. P. Tanana, Theory of Linear Ill-Posed Problems and Its Applications (Nauka, Moscow, 1978; VSP, Utrecht, 2002).

3. V. V. Vasin and A. L. Ageev, Ill-Posed Problems with A Priori Information (VSP, Utrecht, 1995).

4. A. L. Ageev and T. V. Antonova, Izv. Ural'sk. Gos. Univ. 58, 24 (2008).

5. G. Winkler, O. Wittich, V. Liebscher, and A. Kempe, Jahresber. Deutsch. Math.-Verein. 107 (2), 57 (2005).

6. V. S. Sizikov, Mathematical Methods of Processing the Results of Measurements (Politekhnika, St. Petersburg, 2001) [in Russian].

7. S. Mallat, A Wavelet Tour of Signal Processing (Academic, New York, 1999; Mir, Moscow, 2005).

8. Ya. A. Furman, A. V. Krevetskii, A. K. Peredreev, et al., Introduction to Contour Analysis and Its Applications to Image and Signal Processing (Fizmatlit, Moscow, 2002) [in Russian].

9. J. Gilles and Y. Meyer, IEEE Trans. Image Process. 19 (11), 2793 (2010).

10. C. G. M. Oudshoorn, Bernoulli 4 (1), 15 (1998).

11. A. P. Korostelev, Teor. Veroyatnost. i Primenen. 32 (4), 796 (1987).

12. A. L. Ageev and T. V. Antonova, Russian Math. (Iz. VUZ) 51 (11), 1 (2007).

13. T. V. Antonova, Russian Math. (Iz. VUZ) 45 (7), 63 (2001).

14. T. V. Antonova, J. Inverse Ill-Posed Probl. 10 (2), 113 (2002).

15. T. V. Antonova, Proc. Steklov Inst. Math., Suppl. 1, S145 (2002).

16. T. V. Antonova, Sib. Zh. Vychisl. Mat. 13 (4), 375 (2010).

17. A. L. Ageev and T. V. Antonova, Comput. Math. Math. Phys. 48 (8), 1284 (2008).

18. I. P. Natanson, Theory of Functions of a Real Variable (Nauka, Moscow, 1974; Ungar, New York, 1955).

19. A. L. Ageev and T. V. Antonova, Trudy Inst. Mat. Mekh. UrO RAN 17 (3), 30 (2011).

Translated by M. Deikalova 